Consequently, only the more symmetrical conjugated systems or those which do not give rise to strongly ionic structures will show intense fluorescence.

The increase of the number of 'mobile' electrons, for example, in the series benzene $\rightarrow$ naphthalene $\rightarrow$ anthracene $\rightarrow \ldots . \rightarrow$ graphite, makes them behave increasingly like 'metallic' electrons as regards their optical and electrical behaviour.

We have found that this analogy to a metal can also be applied to some extent to the chemical structure and the reactivity of conjugated ring systems in general. It should be possible to prepare stable positive ions from the more 'metal-like' hydrocarbons by the action of oxidizing agents, and these positive ions should be capable of forming salt-like compounds. Various ionic compounds (bisulphate, perchlorate, etc.) have actually been prepared more recently from graphite by Hofmann ${ }^{1}$ and collaborators by acting on it with an oxidizing agent in the presence of strong acids (sulphuric, perchloric). A certain number of hexagons in the layer planes loose an electron to form a monovalent positive (macro)-ion and this ion combines with $\mathrm{HSO}_{4}^{-}$ or $\mathrm{ClO}_{4}^{-}$ion which goes between the layer planes of the graphite.

In view of the above-mentioned analogies with the metals, there can be no doubt that at least some of the peroxides of highly conjugated hydrocarbons, and in particular graphitic oxide, are essentially of ionic nature. The general formula of these compounds is most probably [(Hydrocarbon +$\left.) \mathrm{O}_{2}^{-}\right]$. They are sometimes formed in a thermal reaction as in the case of triphenyl methyl ${ }^{2}$, giving $\left[\left(\mathrm{C}_{6} \mathrm{H}_{5}\right)_{3} \mathrm{C}+. \mathrm{O}_{2}^{-}\right]$. In the case of the diamagnetic hydrocarbons (for example, rubrene ${ }^{3}$ or $3: 10$ dimethyl $1: 2$ benzanthracene ${ }^{4}$, the peroxide is only formed under the influence of light, which decreases the ionization potential in the excited state and thus helps the formation of a positive ion.

The existence of $\mathrm{O}_{2}^{-}$ion in peroxides like $\mathrm{KO}_{2}$ has been shown by Pauling and Neuman ${ }^{5}$ and its electronic structure has been discussed in detail ${ }^{6}$. Graphitic oxide should then be considered as a salt similar to the other graphite salts where the anion $\mathrm{HSO}_{4}^{-}$or $\mathrm{ClO}_{4}^{-}$is now replaced by $\mathrm{O}_{2}^{-}$(or possibly partly by $\mathrm{HO}_{2}^{-}$) (see diagram). This structure, of course, only refers to the 'active' oxygen in graphitic oxide. The rest of the oxygen is present partly in the form of $\mathrm{OH}$ groups (possibly $\mathrm{OH}$-ions) or as molecular water.

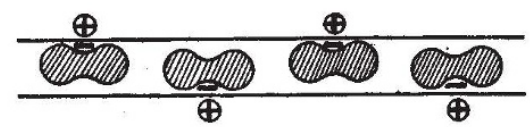

\begin{abstract}
SCHEMATIC REPRESENTATION OF THE STRUCTURE OF GRAPHITIC OXIDE (SHADED PARTS REPRESENT $\mathrm{O}_{2}^{-}$IONS BETWEEN THE LAYER PLANES OF THE GRAPHITE).
\end{abstract}

The ionic nature of graphitic oxide is also confirmed by the observed distance of layer planes, and by the fact that interplanar swelling can only be effected by polar solvents ${ }^{1}$, a fact which was not understood previously.

The positive ions of graphite can be easily reduced ${ }^{1}$ (reversibly) again to graphite, and this is also true for some of the hydrocarbon-peroxides which are decomposed by light or heat giving the unchanged ring systems.
Conjugated systems are of great biological interest and their biological significance seems to lie in the fact that they can undergo reversible oxidation, as has been made very probable in the case of chlorophyll ${ }^{7}$. The carcinogenic hydrocarbons also belong into the group of fluorescent conjugated ring systems ${ }^{8}$ and it is quite possible that the oxido-reduction of these 'metal-like' hydrocarbon ions, namely,

$$
(\text { Hydrocarbon }+) \underset{\text { oxidation }}{\stackrel{\text { reduction }}{\longleftarrow}} \longrightarrow \text { (Hydrocarbon), }
$$

is an essential factor in causing the abnormal metabolism.

A full account will be published elsewhere.

University of Durham, JOSEPH WEISS. King's College,

Newcastle-on-'Tyne. March 21.

${ }^{1}$ Hofmann, U., Ergeh. exact. Naturwiss., 18, 229 (1939).

'Ziegler, K., and Ewald, L., Ann. Chem., 504, 162 (1933).

${ }^{3}$ cf. Dufraisse, C, Bull. Soc. Chim., (5) 16, $4 \dot{2} 2$ (1939).

- Cook, J. W., Martin, R., Roe, E. M. F., Nature, 143, 1020 (1939).

${ }^{5}$ Neuman, E. W., J. Chem. Phys., 2, 31 (1934).

${ }^{6}$ Pauling, L., J. Amer. Chem. Soc., 53, 1367, 3225 (1931).

? Rabinovitch, E., and Weiss, J., Proc. Roy. Soc., A, 162, 251 (1937).

8. cf. Cook, J. W., NATURE, 145, 335 (1940).

\section{Ionization of Calcium Phytate}

IT has been pointed out by Bruce and Callow ${ }^{1}$, and again recently by Harrison and Mellanby ${ }^{2}$, that the rachitogenic property of cereals may be due to the formation of a sparingly soluble salt of phytic acid and calcium. In this laboratory, investigations on the chemical properties of this compound have shown that sodium phytate can only form an insoluble precipitate when an equivalent or an excess of calcium is added. Under such conditions the filtrates are clear and contain only a trace of phytic acid. When less than half the equivalent quantity of calcium is added, no precipitate is formed and the solution remains clear. The calcium in the solution is not precipitated by the addition of phosphate or oxalate. This indicates complex ion formation, and also that the degree of ionization of free calcium is extremely small. When the amount of calcium is between one half and one equivalent the solution is turbid and difficult to filter.

The magnesium ion reacts with sodium phytate in the same way. When a mixture of calcium and magnesium ions is added in excess of that required by the phytate, magnesium can take the place of the calcium in the precipitate to a certain extent.

Henry Lister Institute of

E. F. YANG.

Medical Research,

1320 Avenue Road, Shanghai.

Jan. 30 .

1 Bruce, H. M., and Callow, R. K., Biochem. J., 28, 517 (1934).

${ }^{2}$ Harrison, D. C., and Mellanby, E., Biochem. J., 33, 1660 (1939).

WITH reference to the above letter, we should like to point out that we showed in a recent paper on phytic acid and the rickets-producing action of cereals $^{1}$, that the greater part of the calcium in a hydrochloric acid extract of oatmeal is, in fact, 